\title{
Novel Coronavirus Disease 2019 (COVID-19): A Focused Clinic Overview
}

\author{
Xinhang $\mathrm{Tu}^{1}$ and Miao $\mathrm{Cui}^{2 *}$ \\ ${ }^{1}$ Department of Internal Medicine, Medstar Health Internal Medicine Residency Program, USA \\ ${ }^{2}$ Department of Pathology, Mount Sinai St. Luke's Roosevelt Hospital Center, USA \\ *Corresponding author: Miao Cui, Department of Pathology, Mount Sinai St. Luke's Roosevelt Hospital Center, USA. \\ To Cite This Article: Xinhang Tu, Miao Cui, Novel Coronavirus Disease 2019 (COVID-19): A Focused Clinic Overview. Am J Biomed Sci \& Res. \\ 2021 - 11(4). AJBSR.MS.ID.001642. DOI: 10.34297/AJBSR.2021.11.001642.
}

Received: 眥 December 12, 2020; Published: 制 January 05, 2021

\begin{abstract}
At the end of 2019, a series of COVID-19 related respiratory infections were initially identified in China. After a few months of spreading via human-to-human transmission, it has been rapidly outbreak over 200 countries worldwide, becoming an emergency of primary international concern and posing a severe threat to public health globally. COVID-19 infection can cause clusters of symptoms from mild influenza-like illness to the severe acute respiratory syndrome. A better understanding of clinical symptoms, risk factors, and clinic progression will benefit disease control. This literature review demonstrates the clinical course of COVID-19, including risk factors, clinical presentation, progression and complication.

Keywords: COVID-19; SARS-CoV-2; Pandemic; Respiratory Tract Infections; Clinical Presentation

Abbreviation: COVID-19: Coronavirus Disease 2019; WHO: World health organization; CDC: Centers for Disease Control and Prevention; SD: Standard deviation; COPD: Chronic Obstructive Lung Disease; ARDS: Acute Respiratory Distress Syndrome; DIC: Disseminated Intravascular Coagulation; URT: Upper Respiratory Tract ; CXR: Chest X-ray; CT: Computed Tomography; PaO2: Partial Pressure of Oxygen; FiO2: Fraction of Inspired Oxygen; ICU: Intensive Care Unit; AST : Aspartate Transaminase; ALT: Alanine Transaminase; LDH: Lactate Dehydrogenase; $\alpha$-HBDH: $\alpha$-Hydroxybutyrate Dehydrogenase; SARS: Severe Acute Respiratory Syndrome; MERS: Middle East Respiratory Syndrome Coronavirus; RT-PCR: Reverse Transcriptase Polymerase Chain Reaction
\end{abstract}

\section{Introduction}

The severe acute respiratory syndrome coronavirus-2 (SARS-CoV-2) is a novel coronavirus in one of the members of the family Coronaviridae and the disease caused by this virus, termed coronavirus disease 2019 (COVID-19) by the world health organization (WHO) [1,2]. An outbreak of a COVID-19 infection has been identified since December 2019 in Hubei, Wuhan, China, firstly [3]. Subsequently, it has rapidly spread throughout the world as a global health emergency, drawn attention from the WHO, and declared a pandemic on March 11, 2020. COVID-19 is a human airborne disease, and its transmission is a potential impact as and the infection rate is rapid [4]. Because of no effective target therapy for COVID-19 currently [5], the clinical treatment mainly focused on reducing clinical symptoms [6]. On the other hand, due to the lack of available diagnostic assays, early screening in populations relies on clinical symptoms [7], especially during the incubation period.
Therefore, a comprehensive summary of clinical symptoms and course is essential for early diagnosis and preventive quarantine in communities and supportive management in clinics.

\section{Risk Factors}

Many clinical studies indicated that patients with certain characteristics are at higher risk of infection with COVID-19 compared with the control group, including elder age, male gender, and chronic comorbidities $[2,8]$. For instance, a report from the Chinese Center for Disease Control and Prevention (CDC) showed $77.8 \%$ of patients are between 30 to 69 years old in 44,672 confirmed infections. Among them, 31.2\% are aged above 60 years old and the fatality rate of the population above 60 years is significantly higher than other age groups. Furthermore, the portion of male patients is slightly higher (51.4\%) [9]. Similarly, a report from the Centers for Disease Control and Prevention (CDC) in the United 
States (US) (https://www.cdc.gov/coronavirus/2019-ncov/coviddata/covidview/index.html) revealed the hospitalization rates in people of 65 years and older are the highest among all age groups. However, the median age of the susceptible population and gender ratio vary with locations. In a study of 1591 infected patients in Italy, the median age of infected patients was 63 years, with $82 \%$ were male [10]. In contrast, the cases of Korea between January, 2020 and April 2020 showed the median age of patient was 45 and male patients accounted for $39.9 \%$ [11].

According to US CDC [12], there is a trend towards an increasingly younger population in the US from May, 2020 to July, 2020. The incidence is increased in the $<80$ years group overall, among which the largest increases are the group of $<30$ years. Furthermore, the median age of COVID-19 cases decreased from 46 years to 37 years from May, 2020 to July, 2020. Similarly, the median age of COVID-19 patients in the Europe decreased from 54 years to 39 years from January-May, 2020 to June-July, 2020. Especially, young people (20s age group) account for the largest proportion (19.5\%). Patients with underlying comorbidities are prone to a higher risk for infection (Table 1). Based on a study with 1099 patients diagnosed with COVID-19 in China [13], about $24 \%$ of patients had at least one coexisting illnesses, including cardiovascular disease, diabetes mellitus, hypertension, chronic lung disease, cancer, chronic kidney disease, obesity, smoking. Patients with chronic comorbidities are found to have a higher case fatality rate. A meta-analysis of 1527 patients [20] showed the proportions of hypertension, cardia-cerebrovascular diseases, and diabetes in patients with COVID-19 were $17.1 \%, 16.4 \%$, and $9.7 \%$, respectively, and incidences were about two folds, three folds, and two folds, respectively, higher in the ICU/severe cases than in their non-ICU/non-severe counterparts. In addition, China CDC Weekly [9] demonstrated patients with comorbid conditions had higher case fatality rates-10.5\% for those with cardiovascular disease, $7.3 \%$ for diabetes, $6.3 \%$ for chronic respiratory disease, $6.0 \%$ for hypertension, and $5.6 \%$ for patients with cancer. In comparison, patients with no comorbidities only had a case fatality rate of $0.9 \%$.

\section{Clinical Presentation}

A study of 1099 patients [13] showed the median incubation period was four days with an interquartile range of 2 to 7 days. Whereas data from the US CDC (https://www.coronavirus.gov/) showed the incubation period varies from 2 to 14 days after exposure to the virus. The initial presentation of infected patients can be either symptomatic or asymptomatic. Asymptomatic infections refer to patients diagnosed with positive detection of the nucleic acid of SARS-CoV-2 by reverse transcriptase-polymerase chain reaction (RT-PCR), with no typical clinical symptoms or signs, and no apparent abnormalities in images, such as pulmonary computed tomography (CT). Although the percentage of asymptomatic population varies among different locations and settings, it seems to be more prevalent in areas outside of China and on certain cruises [21]. The clinical presentation spectrum ranges from mild to critical among those who are symptomatic. Data from China CDC [22] revealed that $81 \%$ of 44,415 COVID-19 positive patients presented mild pneumonia or non-pneumonia, $14 \%$ patients had severe disease with symptoms of dyspnea, respiratory frequency $\geq 30 /$ minute, blood oxygen saturation $\leq 93 \%, \mathrm{PaO}_{2} / \mathrm{FiO}_{2}$ ratio $<300$, and/or lung infiltrates $>50 \%$ within $24-48$ hours, and $5 \%$ developed critical disease with presentations of respiratory failure, septic shock, and/or multiple organ dysfunction/failure.

The early stage of its clinical course is similar to common viral respiratory infections, presenting with fever, sore throat, fatigue and cough coupled with recent exposure [23], but compared with others, it can rapidly progress to dyspnea within several days after the initial infection. Based on the current published studies (Table 2 ), the most common typical symptom is fever overall. $43.8 \%$ of all patients reported fever on admission, and $88.7 \%$ developed fever during hospitalization. The following most common symptoms are dry cough [13] and shortness of breath [14]. Besides, gastrointestinal symptoms were not a common finding in the early stage according to clinical observation in China, but they were sometimes found to be the only presenting symptoms based on later reports [24]. Furthermore, anosmia and dysgeusia were more common in Europe and North America than in Asia.

\section{Imaging Findings}

Chest X-rays (CXR) and chest CT are well-known standard diagnostic tools for pulmonary diseases and upper respiratory tract (URT) infections in the clinic. A clinical study involving 1014 cases in the Wuhan [25] showed $97 \%$ of COVID-19 positive patients confirmed by RT-PCR have abnormal chest CT findings. The most common patterns of COVID-19 pneumonia are bilateral patchy shadowing on CXR, with ground-glass opacity (56.4\%) and bilateral patchy shadowing (51.8\%) on the chest CT. However, both CXR and chest CTs can be negative initially or in mild disease $[13,26]$.

\section{Progression}

The clinical course is mainly progress of resolving COVID-19 related infections and secondary systemic inflammatory response [13]. The median time from illness onset to hospital admission is seven days, the median length of hospitalization ranges between 11-12 days, and the ICU admission rate is about 5-26\% [16]. The median time from illness onset to ICU admission is 12 days, from symptom onset to acute respiratory distress syndrome ARDS varies from 7.5 to 12 days, and from illness onset to death or discharge is about 21 days [15]. Furthermore, certain patients can progress to refractory patients who do not reach obvious clinical and radiological remission within 10 days of hospitalization. Aging and male patients and those with comorbidities were more prone to progress to refractory cases [17]. The mortality rate varies in 
different groups and locations [27]. Generally, it is higher in elderly. Heretofore, case fatality rate is $1.9 \%$ in United States and $5.0 \%$ in China from the mortality analyses by the Coronavirus Resource Center, Johns Hopkins University of Medicine on 12/12/2020 (https://coronavirus.jhu.edu/data/mortality) Complications [28] due to severe inflammatory reaction can impact multiple organs, including septic shock, coagulopathy, acute respiratory distress syndrome (ARDS), and end-organ injury (Table 3). Among them, ARDS is one of the most common complications, which can be developed rapidly over a short period. Notably, liver injuries tend to be more prevalent in COVID-19 patients, especially in patients with severe disease, compared with non-COVID-19 pneumonia [29]. Liver injury secondary to COVID-19 infection usually manifests with abnormal hepatic panel results, including increased lactate dehydrogenase (LDH), bilirubin, aspartate transaminase (AST), alanine transaminase (ALT), and $\alpha$-Hydroxybutyrate dehydrogenase $(\alpha-\mathrm{HBDH})$. The abnormal laboratory tests could help to differentiate and evaluate the progress of Covid-19 pneumonia [24].

\section{Conclusion}

The outbreak of emerging severe acute respiratory infections in humans caused by COVID-19 has spread to a global pandemic and become a global health concern [29, 30]. Although the initial symptoms and complications are similar to severe acute respiratory syndrome (SARS) in 2003 and middle east respiratory syndrome coronavirus (MERS) in 2012, the presentation of COVID-19 is less severe with a lower fatality rate overall but a stronger ability to spread in communities than MERS and SARS [31]. However, COVID-19 patients with chronic comorbidities are found to have a higher case fatality rate. Liver injuries are more prevalent in severe COVID-19 cases, varying from transient mild abnormal blood tests to severe liver damage. Since the COVID-19 outbreak is still a significant challenge for clinicians, we urge clinicians to continue reporting their data to understand and control this novel virus better.

\section{Conflicts of Interest}

All authors declare that there is no conflict of interest regarding the publication of this article.

\section{References}

1. Kakodkar P, Kaka N, Baig MN (2020) A Comprehensive Literature Review on the Clinical Presentation, and Management of the Pandemic Coronavirus Disease 2019 (COVID-19). Cureus 12(4): 7560.

2. Sohrabi C, Alsafi Z, O'Neill N, Mehdi Khan, Ahmed Kerwan, et al. (2020) World Health Organization declares global emergency: A review of the 2019 novel coronavirus (COVID-19) Int J Surg 76: 71-76.

3. Jin Y, Yang H, Ji W, Wu W, Chen S, et al. (2020) Virology, Epidemiology, Pathogenesis, and Control of COVID-19. Viruses 12(4): 372.
4. Miller R, Englund K (2020) Clinical presentation and course of COVID-19. Cleve Clin J Med 87(7): 384-388.

5. Zhai P, Ding Y, Wu X, Long J, Zhong Y, et al. (2020). The epidemiology, diagnosis and treatment of COVID-19. Int J Antimicrob Agents 55(5):105955.

6. Thevarajan I, Buising KL, Cowie BC (2020) Clinical presentation and management of COVID-19. Med J Aust 213(3):134-139.

7. Parasher A (2020) COVID-19: Current understanding of its pathophysiology, clinical presentation and treatment. Postgrad Med J.

8. Harapan H, Itoh N, Yufika A, Wira Winardi , Synat Keam, et al. (2020) Coronavirus disease 2019 (COVID-19): A literature review. J Infect Public Health 13(5): 667-673.

9. Zhonghua Liu Xing Bing Xue Za Zhi (2020) The epidemiological characteristics of an outbreak of 2019 novel coronavirus diseases (COVID-19) in China 41(2):145-151.

10. Grasselli G, Zangrillo A, Zanella A, Massimo Antonelli, Luca Cabrini, et al. (2020) Baseline Characteristics and Outcomes of 1591 Patients Infected With SARS-CoV-2 Admitted to ICUs of the Lombardy Region, Italy. JAMA 323(16): 1574-1581.

11. Jung CY, Park H, Kim DW, Choi YJ, Kim SW, et al. (2020) Clinical Characteristics of Asymptomatic Patients with COVID-19: A Nationwide Cohort Study in South Korea. Int J Infect Dis 99: 266-268.

12. Boehmer TK, Devies J, Caruso E, Van Santen KL, Tang S, et al. (2020) Changing Age Distribution of the COVID-19 Pandemic - United States, May-August 2020. MMWR Morb Mortal Wkly Rep 69(39): 1404-1409.

13. Guan WJ, Ni ZY, Hu Y, Liang WH, Ou CQ et al. (2020) China Medical Treatment Expert Group for Covid-19. Clinical Characteristics of Coronavirus Disease 2019 in China. N Engl J Med 382(18): 1708-1720.

14. Tian S, Hu N, Lou J, Chen K, Kang X, et al. (2020) Characteristics of COVID-19 infection in Beijing. J Infect 80(4): 401-406.

15. Wang D, Yin Y, Hu C, Liu X, Zhang X, et al. (2020) Clinical course and outcome of 107 patients infected with the novel coronavirus, SARSCoV-2, discharged from two hospitals in Wuhan, China. Crit Care 24(1): 188.

16. Zhou F, Yu T, Du R, Fan G, Liu Y, et al. (2020) Clinical course and risk factors for mortality of adult inpatients with COVID-19 in Wuhan, China: a retrospective cohort study. Lancet 395(10229):1054-1062.

17. Mo P, Xing Y, Xiao Y, Deng L, Zhao Q, et al. (2020) Clinical characteristics of refractory COVID-19 pneumonia in Wuhan, China. Clin Infect Dis ciaa 270 .

18. Aggarwal S, Garcia-Telles N, Aggarwal G, Lavie C, Lippi G, et al. (2020) Clinical features, laboratory characteristics, and outcomes of patients hospitalized with coronavirus disease 2019 (COVID-19): Early report from the United States. Diagnosis (Berl) 7(2): 91-96.

19. Israelsen SB, Kristiansen KT, Hindsberger B, Ulrik CS, Andersen O, et al. (2020) Characteristics of patients with COVID-19 pneumonia at Hvidovre Hospital, March-April 2020. Dan Med J 67(6): A05200313.

20. Li B, Yang J, Zhao F, Zhi L, Wang X, et al. (2020) Prevalence and impact of cardiovascular metabolic diseases on COVID-19 in China. Clin Res Cardiol 109(5): 531-538.

21. Gao Z, Xu Y, Sun C, Wang X, Guo Y, et al. (2020) A Systematic Review of Asymptomatic Infections with COVID-19. J Microbiol Immunol Infect.

22. Wu Z, McGoogan JM. (2020) Characteristics of and Important Lessons From the Coronavirus Disease 2019 (COVID-19) Outbreak in China: Summary of a Report of 72314 Cases From the Chinese Center for Disease Control and Prevention. JAMA 323(13): 1239-1242. 
23. Jiang F, Deng L, Zhang L, Cai Y, Cheung CW, et al. (2020) Review of the Clinical Characteristics of Coronavirus Disease 2019 (COVID-19). J Gen Intern Med 35(5): 1545-1549.

24. Wong SH, Lui RN, Sung JJ. (2020) Covid-19 and the digestive system. J Gastroenterol Hepatol 35(5): 744-748.

25. Ai T, Yang Z, Hou H, Zhan C, Chen C, et al. (2020) Correlation of Chest CT and RT-PCR Testing for Coronavirus Disease 2019 (COVID-19) in China: A Report of 1014 Cases. Radiology 296(2): 32-40.

26. Shi H, Han X, Jiang N, Cao Y, Alwalid O, et al. (2020) Radiological findings from 81 patients with COVID-19 pneumonia in Wuhan, China: a descriptive study. Lancet Infect Dis 20(4): 425-434.

27. Du RH, Liang LR, Yang CQ Wang W, Cao TZ, et al. (2020) Predictors of mortality for patients with COVID-19 pneumonia caused by SARS-CoV-2: a prospective cohort study. Eur Respir J 55(5): 2000524.
28. Singhal T. (2020) A Review of Coronavirus Disease-2019 (COVID-19). Indian J Pediatr 87(4): 281-286.

29. Zhao D, Yao F, Wang L, Zheng L, Gao Y, et al. (2020) A Comparative Study on the Clinical Features of Coronavirus 2019 (COVID-19) Pneumonia With Other Pneumonias. Clin Infect Dis 71(15):756-761.

30. Kannan S, Shaik Syed Ali P, Sheeza A, Hemalatha K (2020) COVID-19 (Novel Coronavirus 2019) - recent trends. Eur Rev Med Pharmacol Sci 24(4): 2006-2011.

31. Petrosillo N, Viceconte G, Ergonul O, Ippolito G, Petersen E (2020) COVID-19, SARS and MERS: are they closely related? Clin Microbiol Infect 26(6): 729-734. 